\section{Factores que afectan al ambiente educativo en la formación preclínica de medicina según sus docentes}

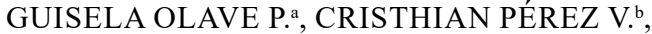 \\ EDUARDO FASCE H.c, LILIANA ORTIZ M. ${ }^{\mathrm{d}}$, NANCY BASTÍAS V.e, \\ CAROLINA MÁRQUEZ U. ${ }^{\mathrm{f}}$, PAULA PARRA P. ${ }^{\mathrm{g}}$, PILAR IBÁÑEZ G. ${ }^{\text {h }}$
}

\section{Factors affecting the educational environment in undergraduate medical schools}

Background: Educational environment has an important effect on the quality oflearning and student satisfaction in medicine. Most of previous studies have been conducted using questionnaires that assess the phenomenon considering overall dimensions, without paying attention to the specific manifestations of this topic, especially those aspects that are related to the protagonists of the learning process: teachers and students. Aim: To describe factors that affect the educational environment in the preclinical Medical formation, according to Medical teachers in Concepción, Chile. Material and Methods: Qualitative study, using the Grounded theory method. Semi-structured interviews were performed to 10 medical teachers in Concepcion, Chile. They were selected by theoretical sampling. Data were analyzed using open coding. Results: Four emerging categories about the factors that affect the learning environment were identified: Personal factors of students, academic factors of students, personal factors of teachers and academic factors of teachers. Conclusions: According to interviewed teachers, both personal factors in teachers and students that promote a positive learning environment are related with an attitude oriented towards others and communication skills. Academic factors are related with the responsible exercise of student and teacher roles and with the promotion of participation in the educational process.

(Rev Med Chile 2016; 144: 1343-1350)

Key words: Education, Medical; Education, Medical, Undergraduate; Mental Health; Stress, Psychological; Students, Medical.

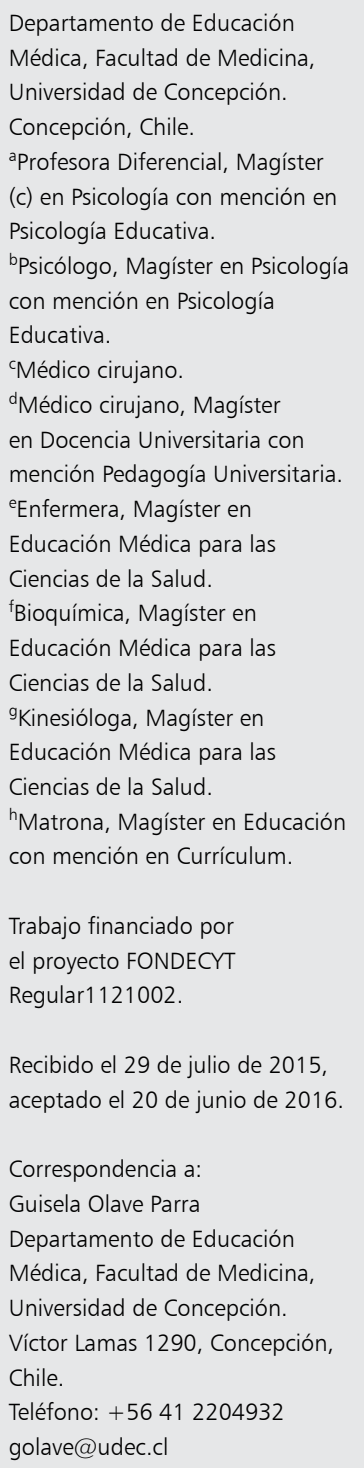

Departamento de Educación Médica, Facultad de Medicina Concepción. (c) en Psicología con mención en Psicología Educativa. con mención en Psicología

Educativa.

'Médico cirujano, Magíster en Docencia Universitaria con eEnfermera, Magíster en Educación Médica para las ncias de la Salud. Ciencias de la Salud.

${ }^{9}$ Kinesióloga, Magíster en Educación Médica para las ias de la Salud.

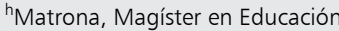

Trabajo financiado por el proyecto FONDECYT Recibido el 29 de julio de 2015 aceptado el 20 de junio de 2016 .

Correspondencia a:

Guisela Olave Parra

Departamento de Educación Dedica, Facultad de Medicin Teléfono: +56412204932 golave@udec.c

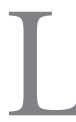

a demanda por un nuevo perfil de médico ha envuelto a la educación médica en profundos $_{\text {cambios }}{ }^{1}$, presionando a las escuelas de medicina para mejorar la calidad de la formación que entregan ${ }^{2}$.

Los esfuerzos por mejorar esta formación han incluido la reformulación de los perfiles de egreso y los programas formativos ${ }^{3-5}$, la capacitación de los docentes ${ }^{6-9}$ y una creciente preocupación por el bienestar de los estudiantes ${ }^{10-12}$ y el ambiente educativo $^{13-20}$.
Entre éstos, el ambiente educativo ha sido identificado como uno de los factores más determinantes de un plan de estudio eficaz ${ }^{17}$, pues correlaciona positivamente con el aprendizaje significativo, el éxito académico y la satisfacción con el programa formativo ${ }^{14,18,19}$.

El ambiente educativo hace referencia a la atmósfera global y característica de la clase y de la facultad ${ }^{15}$, incluyendo el currículum y la infraestructura en que ocurre la formación, pero también sus condiciones físicas, sociales, económicas, 
etc $^{13}$. En este sentido, debe entenderse como un constructo amplio, compuesto por condiciones tanto materiales como inmateriales, incluyendo las relaciones e interacciones de las personas que ahí se encuentran ${ }^{16}$.

En la actualidad, los docentes médicos reconocen los efectos que tiene este constructo tanto a nivel académico y clínico, entendiendo su rol en la adquisición de aptitudes, conocimiento, actitudes $\mathrm{y}$ conductas de los estudiantes de medicina ${ }^{20}$.

Esto mismo ha incrementado el interés por conocer la percepción que los estudiantes tienen en torno a éste ${ }^{18-20}$, a fin de establecer la base para la implementación de modificaciones que permitan mejorar y lograr una mayor eficiencia en las instituciones educativas ${ }^{18}$. Toda vez que la gestión del ambiente educativo en el aula ha adquirido gran relevancia como herramienta para favorecer las condiciones de aprendizaje y el desarrollo de las capacidades, competencias, habilidades y valores de los alumnos ${ }^{21}$.

De ahí que la Federación Mundial de Educación Médica haya declarado que el ambiente educativo debe ser considerado al evaluar los programas de educación médica, dado que la información asociada a éste puede ayudar a potenciar las fortalezas y compensar las debilidades de las instituciones ${ }^{16}$.

De esta forma, en 2007 los investigadores declaraban que la medición del ambiente educativo se había desarrollado en el nivel escolar y universitario, pero no específicamente en las carreras de la salud ${ }^{13}$. Sin embargo, en la actualidad, sólo en carreras de medicina de Chile ya se cuentan varios estudios sobre el tema ${ }^{13-16,19-20}$.

Entre las estrategias que se han desarrollado para aproximarse al ambiente educativo están los cuestionarios, entrevistas, grupos focales $y / u$ observaciones $^{13,16}$, siendo los primeros los más populares en la actualidad ${ }^{19}$.

Entre éstos, uno de los instrumentos más empleados para la medición del ambiente educativo es el DREEM (Dundee Ready Education Environment Measure $)^{19}$, que de acuerdo a la propuesta original de este instrumento, evaluaría cinco dimensiones del ambiente educativo: percepción académica, social, del aprendizaje, de los docentes y de la atmósfera ${ }^{14-15,22-24,26}$. Empero, un estudio en Suecia identificó cinco factores diferentes para el DREEM (aprendizaje y motivación, comunicación, situación psicosocial, organización y pro- gresión de la enseñanza y mala enseñanza ${ }^{25}$ y un análisis factorial en Chile identificó sólo cuatro: percepción académica, de la atmósfera, de los docentes y experiencia académica ${ }^{19}$. En una línea similar, un cuestionario sobre la adaptación a la vida universitaria de estudiantes de seis carreras de la salud de una universidad chilena consideró la adaptación a la vida universitaria, el ambiente afectivo, la calidad de la docencia, el ambiente físico, el cumplimiento de expectativas y las expectativas de futuro ${ }^{27}$.

Sin embargo, estos instrumentos sólo consideran las dimensiones del ambiente educativo pero no sus condicionantes. Principalmente, aquellos aspectos individuales, que más allá de los institucionales y estructurales, impactan la evaluación del espacio de aprendizaje ${ }^{16}$. En atención a esto, el presente estudio busca describirlos factores de los docentes y los estudiantes que afectan el ambiente educativo. Como una primera aproximación se abordará desde la perspectiva de los docentes y específicamente en los niveles preclínicos, donde la formación ocurre mayormente en las instalaciones universitarias.

\section{Metodología}

El presente estudio cualitativo y descriptivo, siguió las directrices de Strauss y Corbin ${ }^{28}$ para la Teoría Fundamentada (Grounded Theory) ${ }^{29}$.

El caso consideraba académicos de Concepción, Chile que dictaran docencia en los primeros dos años de la carrera de medicina, donde las actividades formativas tienen un menor contenido clínico, y que tuvieran más de 2 años de experiencia en esta labor. Fueron seleccionados finalmente 10 docentes mediante muestreo teórico ${ }^{28}$, Tabla 1.

Para la producción de información se realizaron entrevistas temáticas ${ }^{28}$, semiestructuradas, que aunque poseen temas y subtemas predefinidos en un guión, abordan estos en un diálogo flexible que se adapta a cada entrevistado y a cada situación de entrevista, equilibrando la profundidad y la comparabilidad en la información recabada ${ }^{30}$

Las entrevistas las realizó la investigadora principal, previo proceso de consentimiento informado.

El procedimiento fue aprobado por el Comité de Bioética de la Facultad de Medicina de la Universidad ejecutora del estudio. 
Tabla 1. Descripción de los docentes participantes en el estudio

\begin{tabular}{|cllllll|}
\hline Sujeto & Sexo & Edad & Profesión & $\begin{array}{c}\text { Ejercicio } \\
\text { docente }\end{array}$ & $\begin{array}{c}\text { Postgrado } \\
\text { disciplinar }\end{array}$ & $\begin{array}{c}\text { Postgrado en } \\
\text { pedagogía }\end{array}$ \\
\hline I & Mujer & 40 a 44 años & Médico & 14 años & No & Sí, Magíster \\
\hline II & Mujer & 40 a 44 años & Médico & 6 años & Sí, Magíster & Sí, Magíster \\
\hline III & Mujer & 60 a 64 años & Enfermera & 12 años & No & Sí, Magíster \\
\hline IV & Mujer & 35 a 39 años & Bioquímico & 11 años & No & Sí, Magíster \\
\hline V & Mujer & 35 a 39 años & Enfermera & 8 años & Sí, Magíster & No \\
\hline VI & Mujer & 50 a 54 años & Enfermera & 24 años & No & Sí, Magíster \\
\hline VII & Mujer & 55 a 59 años & Ingeniero & 15 años & No & Sí, Magíster \\
\hline VIII & Mujer & 25 a 29 años & Médico & 3 años & No & No \\
\hline IX & Mujer & 40 a 44 años & Médico & 10 años & No & Sí, Magíster \\
\hline X & Hombre & 35 a 39 años & Médico & 13 años & No & No \\
\hline
\end{tabular}

El procesamiento de los datos se hizo mediante codificación abierta, proceso de identificación inductiva de categorías, las que luego son descritas a partir de las propiedades y dimensiones que emergen de los datos ${ }^{28}$. Se realizó triangulación entre analistas.

\section{Resultados}

Desde la codificación abierta, se identificaron cuatro categorías sobre los factores de los estudiantes (Tabla 2) y de los académicos (Tabla 3) que, según estos últimos, influyen en el ambiente educativo. Las subcategorías que las constituyen se describen a continuación y son ilustradas mediante viñetas (citas textuales) en sus tablas respectivas. Cada viñeta es acompañada de un número romano que identifica su informante $y$ un número arábigo que indica el párrafo de la entrevista donde se ubicaba.

\section{Factores personales de los estudiantes}

Los docentes manifiestan que uno de los rasgos personales de los estudiantes que influyen negativamente en el ambiente educativo es lo que denominan egocentrismo, reflejado en una acción ensimismada y enfocada sólo en el propio beneficio.

Por el contrario, identifican algunas actitudes prosociales de los estudiantes que favorecen el ambiente, como el respeto hacia los demás, evidenciado en cosas simples como saludar y en otras como no discriminar a los compañeros. Lo anterior, es considerado de importancia pues si existe un clima de respeto dentro del aula, todo funcionaría adecuadamente y los alumnos se sentirían motivados por aprender. Otras actitudes prosociales serían la empatía de los estudiantes, la humildad y tender a actuar de forma ética y con responsabilidad social.

En esta misma línea, cuando los estudiantes son alegres el ambiente mejora: esto ocurre cuando ellos bromean, sonríen, se divierten y se sienten relajados durante la clase.

También afectan el ambiente las habilidades comunicativas de los estudiantes, tales como mirar a los ojos cuando conversan, mantener una postura de escucha, saber debatir adecuadamente, respetar las opiniones de otros, etc.

Por último, la presencia de conductas disruptivas también afectan negativamente el ambiente en el aula. Por ejemplo, que conversen durante la clase, que tengan conductas desafiantes hacia los profesores o usen un vocabulario grosero.

\section{Factores académicos de los estudiantes}

La participación dentro del aula propicia un ambiente positivo. Esto ocurre cuando los estudiantes tienen la disposición a participar, responden las preguntas del docente y son capaces de formular inquietudes a fin de aclarar dudas. Es 
Tabla 2. Factores de los estudiantes que afectan el ambiente educativo

\begin{tabular}{|c|c|c|}
\hline Categoría & Sub-categorías & Viñeta ilustrativa \\
\hline \multirow[t]{5}{*}{$\begin{array}{l}\text { Factores } \\
\text { personales de } \\
\text { los estudiantes }\end{array}$} & Rasgos individuales & $\begin{array}{l}\text { "A las personalidades de los estudiantes, menos involucrados con el grupo } \\
\text { curso en general, menos comprometidos, como más ensimismados, más } \\
\text { egocéntricos, narcisos..." (148) }\end{array}$ \\
\hline & Actitudes prosociales & $\begin{array}{l}\text { "... deberían ser empáticos, o sea deberían ser buenas personas del alma, } \\
\text { deberían tener espíritu de servicio..." (III24) }\end{array}$ \\
\hline & Alegría & $\begin{array}{l}\text { "cuando tú ves que los estudiantes están participando, con sus caritas atentas, } \\
\text { que te sonríen, que te tiran bromas, en general lo pasamos bien todos" (112) }\end{array}$ \\
\hline & $\begin{array}{l}\text { Habilidades de } \\
\text { comunicación }\end{array}$ & $\begin{array}{l}\text { "... respetar las opiniones de los demás, respetar su turno de intervenir cierto } \\
\text { en alguna discusión; obviamente expresarse de manera correcta, adecuada, } \\
\text { sin uso de palabras que no corresponden, cierto, dentro del aula..." (IX 28) }\end{array}$ \\
\hline & Conductas disruptivas & $\begin{array}{l}\text { "... los estudiantes son desafiantes con los profesores. Ya no tienen una ac- } \\
\text { titud de, si por último no están disfrutando, de quedarse callados y aburrirse } \\
\text { solos... sino además de entorpecer la actividad, pese a lo que tú puedas hacer } \\
\text { como para corregir la dinámica" (I28) }\end{array}$ \\
\hline \multirow[t]{4}{*}{$\begin{array}{l}\text { Factores } \\
\text { académicos de } \\
\text { los estudiantes }\end{array}$} & Participación & $\begin{array}{l}\text { "... la buena disposición a participar, a responder si se hacen preguntas, } \\
\text { a aclarar dudas si las tienen, y eso siempre contribuye a que la clase sea } \\
\text { mejor"(II30) }\end{array}$ \\
\hline & Atención & $\begin{array}{l}\text { "... que están atentos, esos son los que logran que el profesor haga mejor su } \\
\text { trabajo. O sea, son condiciones como mínimas, pero que hacen la diferencia } \\
\text { entre un buen grupo y un grupo no tan bueno" (I164) }\end{array}$ \\
\hline & Responsabilidad & $\begin{array}{l}\text { "... que se den cuenta los alumnos que tienen que estar estudiando todos los } \\
\text { días, o sea para estar en medicina uno tiene que estar actualizándose y está } \\
\text { ahí el autoaprendizaje y otras cosas" (III26) }\end{array}$ \\
\hline & Motivación & $\begin{array}{l}\text { "... un alumno que no esté motivado o que no tenga intención de participar } \\
\text { positivamente en una actividad también podría ser un alumno que deteriora } \\
\text { el clima" (V40) }\end{array}$ \\
\hline
\end{tabular}

decir, cuando colaboran activamente dentro del aula permitiendo así la construcción del conocimiento entre todos.

Por el contrario, el ambiente se vuelve incómodo para el docente si, en lugar de mantenerse atentos, los estudiantes no se concentran y presentan actitudes inapropiadas dentro del aula, tales como, conversar, interrumpir, reírse sin un chiste colectivo o estar preocupados constantemente de aparatos electrónicos.

Un tercer factor que destacan los docentes es la responsabilidad, que se manifiesta en que los estudiantes estudien, se preparen y sean buenos alumnos, lo que ayuda a que se vuelvan aprendices autónomos, logrando el autoaprendizaje y la autoevaluación. También se refleja en tener hábitos de estudios, es decir, que estudien sistemáticamente, organicen sus actividades, y sean perseverantes. Y por último, se traduce en cumplir con los compromisos establecidos (p. e. presentación de trabajos, certámenes, etc.) y mostrar una actitud positiva frente al aprendizaje. Por el contrario, el ambiente se ve afectado cuando no traen los materiales solicitados, no cumplen con las lecturas previas y se justifican constantemente por no cumplir.

Los tres factores antes mencionados estarían mediados por la motivación. Se considera vital que los estudiantes estén motivados por la carrera y por las clases, debido a que la motivación facilita e incentiva el aprendizaje. El caso opuesto se evidencia en estudiantes que se mantienen con disgusto y aburrimiento.

\section{Factores personales de los docentes}

En relación a las características personales de los docentes que influyen positivamente en el ambiente dentro del aula, se encuentra el que sean 
Tabla 3. Factores de los docentes que afectan el ambiente educativo

\begin{tabular}{|c|c|c|}
\hline Categoría & Sub-categorías & Viñeta ilustrativa \\
\hline \multirow{4}{*}{$\begin{array}{l}\text { Factores per- } \\
\text { sonales de los } \\
\text { docentes }\end{array}$} & Actitud empática & $\begin{array}{l}\text { "... de ser empáticos, de dar espacios y dar tiempos. Yo creo que al alumno hay } \\
\text { que darle el espacio físico de sentarnos aquí, conversar y dar el tiempo" (II60) }\end{array}$ \\
\hline & Relación respetuosa & $\begin{array}{l}\text { "El respetar a los estudiantes (...). Porque veo en el medio que los docentes } \\
\text { en general no respetan mucho a los otros, no solamente a los estudiantes, } \\
\text { sino que incluso a los pares" (I140) }\end{array}$ \\
\hline & $\begin{array}{l}\text { Instancias de } \\
\text { comunicación }\end{array}$ & $\begin{array}{l}\text { "...docentes que no permiten que el alumno conteste. Hay docentes que } \\
\text { quizás no les gusta que el alumno sepa alguna respuesta y entonces hablan y } \\
\text { no generan ni siquiera preguntas entre ellos. Yo creo que esos son docentes } \\
\text { que podrían no facilitar un buen clima" (V42) }\end{array}$ \\
\hline & $\begin{array}{l}\text { Habilidad para } \\
\text { manejar situaciones } \\
\text { difíciles }\end{array}$ & $\begin{array}{l}\text { "... la docencia en sí misma es súper estresante a veces, y a eso le sumas las } \\
\text { cosas personales... tú vida. Entonces hay que saber lidiar con ambas. Y no } \\
\text { desquitarte con el alumno. Primero, porque no es justo. Y luego, porque un } \\
\text { solo arranque tuyo puede dañar la relación con ellos para siempre" (X76) }\end{array}$ \\
\hline \multirow[t]{4}{*}{$\begin{array}{l}\text { Factores aca- } \\
\text { démicos de los } \\
\text { docentes }\end{array}$} & $\begin{array}{l}\text { Desempeño respon- } \\
\text { sable }\end{array}$ & $\begin{array}{l}\text { "... un docente de la carrera de medicina (...) yo creo que tiene que ser un } \\
\text { modelo a seguir en todo aspecto, en la responsabilidad, en la presencia, en } \\
\text { la actitud, en tener un buen trato con los alumnos, en ser un buen profesio- } \\
\text { nal..." (V62) }\end{array}$ \\
\hline & $\begin{array}{l}\text { Metodologías } \\
\text { participativas }\end{array}$ & $\begin{array}{l}\text { "...no quedarse estancado no cierto, con técnicas o metodologías que ya han } \\
\text { pasado y también el tema disciplinario, que se actualice disciplinariamente } \\
\text { también..." (IX22) }\end{array}$ \\
\hline & Docencia motivadora & $\begin{array}{l}\text { "...yo creo que un docente motivador va a generar un buen clima en el aula, } \\
\text { donde sea y en el fondo en el año que sea..." (V106) }\end{array}$ \\
\hline & $\begin{array}{l}\text { Preparación } \\
\text { pedagógica }\end{array}$ & $\begin{array}{l}\text { "Ehh, hay diploma o diplomados o magíster en la educación o en educación } \\
\text { médica (...), Creo que eso mejora enormemente. O sea, muchos docentes } \\
\text { dicen: 'yo antes hacía esto y estaba pésimo, pero, mira, ahora lo cambie' (...) } \\
\text { Eso va mejorando en alguna medida lo que es el clima de aula: lo que es la } \\
\text { relación con el alumno, mejor aprendizaje" (IV80) }\end{array}$ \\
\hline
\end{tabular}

empáticos con los estudiantes, ya que esto aumenta la motivación y el aprendizaje del alumnado. Lo anterior estaría asociado conexhibir valores universales como el respeto, la preocupación por los otros y el altruismo.

También consideran fundamentales el respeto y la cordialidad en la relación con los estudiantes, pues facilita que se establezca un clima de confianza, propicia el diálogo entre ambos, y permite que los docentes puedan orientar y apoyar a los alumnos. Lo opuesto lo caracterizan aquellos docentes que no respetan a los estudiantes ni a sus pares y los docentes autoritarios que critican agresivamente al alumnado.

Un tercer elemento, alude a que los docentes generen instancias de comunicación adecuada, donde se propicie el diálogo con los estudiantes. El no otorgar estos espacios, o no respetarlos (p.e. no respetar los horarios de atención o correos electrónicos) produce un distanciamiento en la relación docente-alumno.

El cuarto elemento tiene que ver con la habilidad de los docentes para enfrentar situaciones difíciles. Algunas situaciones tales como la acumulación de eventos negativos en casa, problemas externos al trabajo, el tener poco tiempo para preparar las clases y la falta de tiempo para reflexionar sobre la vida en general, producen que los docentes reacciones de manera poco asertiva con los estudiantes, afectando negativamente el ambiente dentro del aula.

\section{Factores académicos de los docentes}

Entre estos factores, los docentes destacan el desempeño responsable, que se refleja en la presentación personal, el cumplimiento oportuno y eficiente de las labores académicas, el respeto por la labor que desempeñan, el compromiso con la 
disciplina y el espíritu de servicio, central, pues medicina trabaja directamente con personas. Lamentablemente, los docentes acusan que estas características se están perdiendo.

Otro factor que favorece un buen ambiente es el uso metodologías participativas, donde los docentes involucran a los estudiantes haciéndolos parte activa de su propio aprendizaje, permitiendo que desarrollen la autorregulación y favoreciendo aprendizajes significativos. Esto implicaría a dejar atrás técnicas o metodologías que consideran del pasado, como; las clases tradicionales o magistrales que no permitirían la reflexión, ni la participación de los estudiantes.

Una docencia motivadora es otro factor necesario para un ambiente positivo: Que el docente entusiasme con lo que está enseñando, que desee enseñar y que traduzca esto en actividades motivadoras, entretenidas y significativas a los estudiantes.

Por último, se destaca la necesidad de preparación en temáticas pedagógicas por parte de los docentes para cumplir con lo anterior, ya que permite la implementación de nuevas metodologías y estrategias innovadoras. Esto favorece clases más dinámicas y motivadoras para los estudiantes.

\section{Discusión}

Cuestionarios, como el DREEM, han hecho un aporte significativo en la evaluación del ambiente educativo, permitiendo evaluar su relación con aspectos institucionales ${ }^{14-15}$, formativos ${ }^{15,20}$, del desempeño académico ${ }^{18}$, etc. Sin embargo, estos instrumentos sólo identifican factores globales del fenómeno ${ }^{19,25-26}$, de forma que el análisis de sus manifestaciones específicas pasa a un segundo plano.

En este sentido, aunque el ambiente es construido en parte por las interacciones sociales que ocurren en el proceso educativo ${ }^{16}$, y pese a que los distintos estudios del DREEM han relevado la figura del docente y las relaciones entre estudiantes como factores a considerar ${ }^{14,15,19,22-24,26}$, son pocos los estudios que han abordado de manera detallada cuáles son los aspectos específicos de docentes y estudiantes que afectan el ambiente de las carreras de medicina. Este es el propósito del presente estudio, específicamente en los niveles iniciales de la formación, cuando ésta ocurre prioritariamente en espacio universitario.
Los resultados, tanto sobre docentes como sobre alumnos, muestran que uno de los aspectos más valorados es el comportamiento orientado hacia al otro, que se caracteriza por la disposición y capacidad para establecer relaciones empáticas, respetuosas y preocupadas de los demás. Lo anterior generaría un escenario adecuado para que docentes y alumnos interactúen positivamente, beneficiando el ambiente educativo.

Sin embargo, no podemos esperar que lo anterior simplemente ocurra. Esto, pues incluye características propias del profesionalismo médico ${ }^{31}$, que por consenso social debe ser promovido en la formación médica ${ }^{1} y$, por compromiso institucional, es parte de la mayoría de los perfiles de egreso de las escuelas de medicina chilenas ${ }^{3,5}$.

$\mathrm{Al}$ ser una meta deseable de la educación médica $^{1,3,5,31}$, y, tal como lo identifica este estudio, al favorecer un ambiente educativo positivo, es que el pregrado mismo debería comprometerse en la promoción deliberada y organizada de estas actitudes. Esto implica, tanto implementar actividades distintivas para desarrollarlas en estudiantes, como capacitar a los docentes para que lo hagan. En efecto, en un estudio en docentes de carreras de la salud chilenos, estos valoraban ampliamente la capacitación en estrategias para gestionar el ambiente educativo9.

Algo similar ocurre con la comunicación, que muestra que no basta con tener actitudes orientadas hacia los demás, sino que es necesario tener habilidades comunicativas y de autorregulación para hacerlo. $\mathrm{Y}$ estos aspectos también están presentes en los perfiles de egresos de las escuelas de medicina ${ }^{3,5}$, que subrayan el rol de la comunicación.

Empero, aunque estos perfiles de egreso comprometen qué competencias se espera formaren los estudiantes, es necesario formarlas primero en los profesores, que serán los encargados de su enseñanza directa $y$, además, de su modelado conciente e inconciente.

Por último, respecto a los factores que favorecen un ambiente positivo, el primero está asociados al ejercicio responsable del rol de los estudiantes y los docentes, lo que implica ejercer comprometida y responsablemente sus respectivas funciones. En este sentido, la complementariedad entre ambos roles sólo sería posible cuando uno de ellos puede hacer su parte sabiendo que el otro va a cumplir diligentemente con lo que se espera de 
él. Esto clarifica el escenario de trabajo y favorece un ambiente grato.

El segundo factor, está asociados a promover la participación. Esto implica desde el alumno mostrarse atento, participativo y motivado en clases, $y$, desde el docente, favorecer dicha participación a través de metodologías que incluyan y entusiasmen. En esta línea, la preparación pedagógica de los docentes es clave $e^{6-9}$. Esto no implica que la participación del estudiante sea sólo una consecuencia de la calidad de la docencia. También existe responsabilidad de los estudiantesde ser capaces de regular su propio comportamiento y emocionalidad para mantener un alto compromiso y motivación. Esto, pues aunque la motivación es afectada por decisiones pedagógicas del docente, siguen siendo una característica individual: del alumno. Instalar el peso de este factor sólo en el docente, además de ser contraproducente, despoja al alumno de su carácter de agente y mina su participación en un proceso formativo que debe protagonizar.

\section{Referencias}

1. Wojtczak A. Profesionalismo médico; una problemática global. Revista de Educación Médica 2006; 2: 144-5.

2. Castellanos M, Cañellas J, Mir I, Águila M. Evaluación de la calidad en la Educación Médica. Perspectivas en el contexto del Policlínico Universitario. Medisur 2008; 6 (2): 161-9.

3. Parada M, Romero MI, Moraga F. Perfiles de egreso de las carreras de medicina en Chile. Rev Med Chile 2015; 143 (4): 512-9.

4. Martinic S, Moreno R, Müller M, Pimentel F, Rittershaussen S, Calderón M, et al. Análisis comparativo del componente de práctica en el currículo de formación profesional de médicos y profesores en la Pontificia Universidad Católica de Chile. Estud pedagóg 2014; 40 (1): 179-96.

5. Ortiz L, Gajardo J. Propuesta de competencias genéricas para el perfil de egreso del médico cirujano de la Universidad de Concepción, Chile. Educ Med Super 2014; 28(3): 424-35.

6. Triviño X, Sirhan M, Moore P, Montero L. Impacto de un programa de formación en docencia en una escuela de medicina. Rev Med Chile 2011; 139: 1508-15.

7. Montero L, Triviño X, Sirhan M, Moore P, Leiva L. Barreras para la formación en docencia de los profesores de medicina: una aproximación cualitativa. Rev Med Chile 2012; 140: 695-702.
8. Sirhan M, Triviño X. Evaluación de una experiencia de capacitación en planificación educacional para directores de programas de las especialidades médicas. Rev Med Chile 2012; 140: 530-7.

9. Pérez C, Fasce E, Coloma K, Vaccarezza G, Ortega J. Percepción de académicos de carreras de la salud de Chile sobre el perfeccionamiento docente. Rev Med Chile 2013; 141: 787-92.

10. Pérez C, Bonnefoy C, Cabrera A, Peine S, Macaya K, Baquedano, $M$ et al. Problemas de salud mental en alumnos universitarios de primer año de Concepción, Chile. An Psicol 2012; 28 (3): 797-804.

11. Dyrbye L, Thomas M, Harper W, Massie S, et al. The learning environment and medical student burnout: a multicentre study. Med Educ 2009: 43: 274-82.

12. Gómez P, Pérez C, Parra P, Ortiz L, Matus B, McColl $\mathrm{P}$, et al. Relación entre el engagement, burnout y el rendimiento académico en alumnos de primer año de medicina. Rev Med Chile 2015; 143 (7): 937-44.

13. Riquelme A, Fuentes G, Jeria A, Méndez I, Aranís C, Larios G, et al. Ambiente educacional y calidad de la docencia en la escuela de medicina. ARS Médica 2007; 15: 1.

14. Herrera C, Pacheco J, Rosso F, Cisterna C, Aichele D, Becker S, et al. Evaluación del ambiente educacional pre-clínico en seis Escuelas de Medicina en Chile. Rev Med Chile 2010; 138: 677-84.

15. Díaz-Véliz G, Mora S, Bianchi R, Gargiulo P, Terán C, Gorena D, et al. Percepción de los estudiantes de medicina del ambiente educativo en una facultad con currículo tradicional (UCH-Chile) y otra con currículo basado en problemas (UNC-Argentina). Educ Med 2011; 14 (1): 27-34.

16. Herrera C, Olivos T, Román J, Larraín A, Pizarro M, Solís N, et al. Evaluación del ambiente educacional en programas de especialización médica. Rev Med Chile 2012; 12: 1554-61.

16. Ríos D, Bozzo N, Marchant J, Fernández P. Factores que inciden en el clima de aula universitario. Revista Latinoamericana de Estudios Educativos 2010; 3: 105-26.

17. Omer H. DREEM; dreams of the educational environment as its effect on education result of 11 Medical Faculties of Turkey. Journal of Experimental and Clinical Medicine 2010; 27: 104-8.

18. Aghamolaei T, Fazel I. Medical students` perceptions of the educational environment at an Iranian Medical Sciences University. Revista BMC Medical Education 2010; 10: 1-7.

19. Ortega J, Pérez C, Ortiz L, Fasce E, McColl P, Torres G, et al. Estructura factorial de la escala DREEM en estudiantes de medicina chilenos. Rev Med Chile 2015; 143 (5): 651-7. 
20. Díaz D, Mora S, Escanero J. Análisis del ambiente educacional tras la implementación del Bolonia en la facultad de medicina de la Universidad de Zaragoza, España. Comparación con la facultad de medicina de la Universidad de Chile. FEM 2013; 3: 167-79.

21. Obayo A, Vargas Y, Ponce C. La gestión de ambientes en el aula y el desarrollo de competencias. Contactos 2012; 83: 21-8.

22. Bassaw B, Roff S, Mcaleer S, Roopnarinesingh S, De Lisle J, Teelucksingh S, et al. Students ' perspectives on the educational environment. Med Teach 2003; 25 (5): 522-6.

23. Al-Hazimi A, Al-Hyiani A, Roff S. Perceptions of the educational environment of the medical school in King Abdul Aziz University. Med Teach 2004; 26 (6): 570-3.

24. Carmody D, Jacques A, Denz-penhey H, Puddey, Newnham J. Perceptions by medical students of their educational environment for obstetrics and gynaecology in metropolitan and rural teaching sites. Med Teach 2009; 31: 596-602.

25. Jakobsson U, Danielsen N, Edgren G. Psychometric evaluation of the Dundee Ready Educational Environment
Measure: Swedish version. Med Teach 2011; 33: 267-74. 26. Rotthoff T, Ostapczuk M, De Bruin J, Decking U, Schneider M, Ritz-timme S. Assesing the learning environment of a faculty: Psychometric validation of the German version of the Dundee Ready Education Environment Measure with students and teachers. Med Teach 2011; 33: 624-36.

27. López M, Vivanco Z, Mandiola E. Percepción de los alumnos sobre su primer año de universidad: Facultad de Medicina Universidad de Chile. Educ Med 2006; 9(3): 31-7.

28. Strauss A, Corbin J. Bases de la investigación cualitativa. Universidad de Antioquía. Medellín 2002.

29. Glaser B, Strauss A. The discovery of Grounded Theory. Aldine Transaction. New Jersey 1967.

30. Flick U. Introducción a la investigación cualitativa. Morata. Madrid 2004.

31. Fasce E, Echeverría M, Matus O, Ortiz L, Palacios S, Soto A. Atributos del profesionalismo estimados por estudiantes de medicina y médicos. Análisis mediante el modelo de disponibilidad léxica. Rev Med Chile 2009; 137 (6): 746-52. 